\title{
Discovery of Five Bright Low-Redshift Quasars from ROSAT PSPC Sources ${ }^{1}$
}

\author{
Jianyan Wei, Li Cao, Jiangyao $\mathrm{Hu}$, and Qibin Li \\ Beijing Astronomical Observatory, Chinese Academy of Science, \\ Beijing, 10080, China
}

\begin{abstract}
A program to identify bright $R O S A T$ sources is being carried out with the $216-\mathrm{cm}$ and $60-\mathrm{cm}$ telescopes at Xinglong station. As the result of the first run, five bright low-redshift quasars have been discovered.
\end{abstract}

\section{Observations and Results}

Both ROSAT All-Sky Survey (RASS) and PSPC pointed observations resulted in many unidentified sources. Optical follow-up observations are needed to identify these sources. A sample of bright $R O S A T$ sources was selected from the PSPC pointed observations according to the following criteria: (1) declination $\delta \geq-10^{\circ}$, (2) Galactic latitude $|b| \geq 20^{\circ}$, (3) count rate $\geq 0.05 \mathrm{~s}^{-1}$, (4) angular extent $\leq 6^{\prime \prime},(5)$ no previous identification as AGNs, cataclysmic variables, white dwarfs, or X-ray binaries, and (6) apparent magnitude $13.5 \leq V \leq 16.5$ on the Digital Sky Survey. A total of about 150 sources were obtained. It is expected that stars having active coronae should occupy majority of the sample. Low-redshift AGNs are also expected.

The spectra of 96 sources have been obtained with the 216-cm telescope at Xinglong station. The typical spectral range covered is $3800-8000 \AA$, with resolution $\sim 4.7 \AA$ per pixel. Classification of the spectra shows that 5 of them are quasars. They have no previous identification in the NASA/IPAC Extragalactic Database (NED). Their $V$ magnitudes have been obtained with the $60-\mathrm{cm}$ telescope at Xinglong station. Their redshifts are $z=0.081,0.143,0.165,0.312$, and 0.76 , and magnitudes are $V=15.4,15.6,16.0,16.4,15.7 \mathrm{mag}$, respectively.

Our results show that optical follow-up identifications of ROSAT PSPC sources are productive in discovering bright low-redshift quasars. There are about 140 quasars brighter than $V=16.0 \mathrm{mag}$ in the northern hemisphere, so the optical follow-up identifications of RASS sources will greatly increase the number and space density of known bright low-redshift quasars.

\section{References}

Véron-Cetty, M.-P., \& Véron, P. 1993, ESO Scientific Report, No. 13.

\footnotetext{
${ }^{1}$ This work is supported by the National Natural Science Foundation of China.
} 
Voges, W. 1992, in Proceedings of the ISY Conference 'Space Science', ESA ISY-3, ESA Publications, 9.

Voges, W., et al. 1994, ROSAT NEWS, No.32. 